[Original article]

\title{
Clinical course and background of nasopharyngeal antibiotic-resistant bacteria carriers among preschool children hospitalized for lower respiratory tract infection
}

\author{
Aya Takeyama $^{1)}$, Kenta Suzuki ${ }^{2)}$, Masaki Ito ${ }^{1)}$, Masatoki Sato ${ }^{2)}$, Koichi Hashimoto ${ }^{2)}$, \\ Masahiko Katayose ${ }^{3)}$ and Mitsuaki Hosoya ${ }^{2)}$ \\ ${ }^{1)}$ Department of Pediatrics, Public Soma General Hospital, Fukushima, Japan, ${ }^{2)}$ Department of Pediat- \\ rics, School of Medicine, Fukushima Medical University, Fukushima, Japan, ${ }^{3)}$ Nanohana Kids Clinic, \\ Fukushima, Japan
}

(Received May 21, 2021, accepted October 27, 2021)

\begin{abstract}
We investigated the nasopharyngeal microbiota in preschool patients hospitalized with lower respiratory tract infection to clarify the relationships between culturable nasopharyngeal bacteria and prognosis. From 2016 to 2018, nasopharyngeal culture was performed on inpatients under 6 years of age with a lower respiratory tract infection. Among the 1,056 study patients, 1,046 provided nasopharyngeal samples that yielded positive cultures, yielding 1,676 isolated strains. Streptococcus pneumoniae, Haemophilus influenzae, and Moraxella catarrhalis, were isolated in 25\%, 27\%, and 31\% of the samples, respectively, and were the major causes of respiratory tract infection in these children. The only factor associated with the isolation of antibiotic-resistant strains from the nasopharynx was daycare attendance, which did not affect clinical severity, such as duration of fever and hospitalization. This study demonstrated that resistant bacteria in the nasopharynx did not affect the severity of lower respiratory tract infection and supports the use of narrow-spectrum antimicrobial agents in accordance with published guidelines when initiating therapy for pediatric patients with community-acquired pneumonia.
\end{abstract}

Key words : nasopharyngeal carrier, lower respiratory tract infection, antibiotic-resistant bacteria, clinical severity, antibacterial agents

\section{Introduction}

Lower respiratory tract infection in children is often preceded by a viral upper respiratory tract infection which is accompanied by respiratory epithelium injury, swelling-related airway obstruction, abnormal secretions, and cell debris, because upper respiratory tract infections may spread along the airways ${ }^{1)}$. The disturbance of normal host defense mechanisms and modification of host bacterial flora predisposes affected individuals to secondary bacterial infection ${ }^{1)}$. The nasopharyngeal microbiota may be involved in the onset and/or progression of lower respiratory tract infections through the mechanisms described above ; nevertheless, it is difficult to evaluate the relationship between the nasopharyngeal microbiota and the causal bacteria of lower respiratory tract infections ${ }^{2,3)}$. Washed sputum culture collection is standard procedure for diagnosis of non-intubated children. However, it is difficult to collect sputum in infants and young children; nasopharyngeal swab sample collection is therefore considered non-invasive and clinically relevant.

In the nasopharynges of children with upper or lower respiratory tract infections, Streptococcus pneumoniae, Haemophilus influenzae, and Moraxella

Corresponding author: Aya Takeyama E-mail : ayatake@rondo.ocn.ne.jp

(C)2021 The Fukushima Society of Medical Science. This article is licensed under a Creative Commons [Attribution-NonCommercial-ShareAlike 4.0 International] license.

https://creativecommons.org/licenses/by-nc-sa/4.0/ 
catarrhalis are often detected ${ }^{4,5)}$. S. pneumoniae and $H$. influenzae are becoming increasingly resistant to penicillin and amoxicillin ${ }^{4}$, and the growing likelihood of antibiotic-resistant bacteria must be considered in general clinical practice.

Here, we retrospectively analyzed the medical records of patients under 6 years of age who had been hospitalized and treated for lower respiratory tract infection during the period from 2016 to 2018. We performed nasopharyngeal culture at admission in all patients to examined their bacterial carriage statuses. We also compared the clinical backgrounds of the children from whom any of the three major causative bacteria (S. pneumoniae, $H$. influenzae, and M. catarrhalis) and their resistant strains, or other antibiotic-resistant bacteria were isolated, then evaluated the effects of these pathogens on the patient clinical course.

\section{Materials and methods}

\section{Study design}

Participants in this study were children under 6 years of age who had been admitted to the Department of Pediatrics, Soma General Hospital, with a diagnosis of lower respiratory tract infection during the period from January 2016 to December 2018. Their medical records, including their nasopharyngeal bacterial culture results, were retrospectively evaluated. Bronchitis, bronchopneumonia, and bronchiolitis, all diagnosed based on physical findings and chest X-ray examination results, were regarded as lower respiratory tract infection. Only patients treated at our hospital were included in the study ; children who required ventilatory management (involving transfer to a tertiary hospital) were excluded. The study protocol was approved by the Ethics Committee of Soma General Hospital on December 29, 2017. A document was posted in the hospital describing the details of the study and stating that patients and their families could opt out of the study if they wished.

\section{Specimen collection and detection}

Nasopharyngeal swabs were obtained by trained staff from all patients immediately upon admission. Aerobic culture was performed using blood agar medium and mannitol salt medium ; 5\%-6\% carbon dioxide gas cultures were performed using chocolate medium. Drug susceptibility analysis was performed by using a microliquid dilution meth- od with a microscan panel (Beckman Coulter, Brea, CA, USA) that complied with the 2012 criteria of the Clinical and Laboratory Standards Institute. In accordance with these criteria, S. pneumoniae with a minimum inhibitory concentration (MIC) for penicillin of $\leq 0.06 \mu \mathrm{g} / \mathrm{ml}, 0.12-1.0 \mu \mathrm{g} / \mathrm{ml}$, or $\geq 2.0 \mu \mathrm{g} / \mathrm{ml}$ was defined as penicillin-susceptible $S$. pneumoniae (PSSP), penicillin-intermediately resistant $S$. pneumoniae (PISP), or penicillin-resistant S. pneumoniae (PRSP). H. influenzae was categorized in accordance with the combined results of a $\beta$-lactamase production test and the MIC for ampicillin (ABPC). $\beta$-lactamase-nonproducing, ABPC-susceptible $\mathrm{H}$. influenzae (BLNAS) isolates had an ABPC MIC of $<4 \mu \mathrm{g} / \mathrm{ml} ; \beta$-lactamase-nonproducing, ABPC-resistant $H$. influenzae (BLNAR) isolates had an ABPC MIC of $\geq 4 \mu \mathrm{g} / \mathrm{ml}$; and $\beta$-lactamase producing, ABPC-resistant $H$. influenzae (BLPAR) isolates had an ABPC MIC of $\geq 4 \mu \mathrm{g} / \mathrm{ml}$. S. aureus with an MIC of $\geq 4 \mu \mathrm{g} / \mathrm{ml}$ for oxacillin was defined as methicillin-resistant $S$. aureus (MRSA).

\section{Clinical background of patients}

For each patient, the following clinical background information was obtained from medical records : age, sex, presence of cohabiting siblings, daycare use, underlying diseases (e.g., preterm birth/low birthweight, congenital heart disease, congenital abnormalities, or chromosomal abnormalities), and history of vaccination with the H. influenzae type $\mathrm{b}$ (Hib) vaccine and pneumococcal vaccine (PCV7/PCV13). Information concerning the use of antibacterial drugs at other clinics or at our hospital until immediately before admission was also collected from medical records. For each patient, the following clinical information was obtained: leukocyte count and C-reactive protein (CRP) value from a blood test at admission ; length of hospital stay ; period of post-hospitalization fever ; and administration of antibacterial drugs, oxygen, and/or hydrocortisone during hospitalization.

\section{Statistical analysis}

Statistical analysis was conducted to ascertain the relationships between isolation of any of the three major causative bacteria or antibiotic-resistant bacteria and clinical characteristics (i.e., clinical background and course factors). Univariate analyses were performed using the chi-square test (for categorical variables) or the Mann-Whitney U test (for continuous variables). Logistic regression 
analysis was performed for multivariate analysis ; each factor with a $p$-value of $<0.05$ in univariate analysis was used in the multivariate analysis. Results with a $p$-value of $<0.05$ were considered statistically significant. SPSS Statistics (version 25.0 ; SPSS, Inc., Chicago, IL, USA) was used for statistical analysis.

\section{Results}

\section{Bacteria isolated from the nasopharynx}

In total, 1,056 children under 6 years of age were hospitalized for lower respiratory tract infection between 2016 and 2018. Among the 1,056 patients, 1,046 provided samples that yielded positive nasopharyngeal cultures. Because multiple strains of bacteria were isolated from some patients, 1,676 total strains of bacteria were isolated (Fig. 1). $S$. pneumoniae comprised 401 (25\%) of the isolated

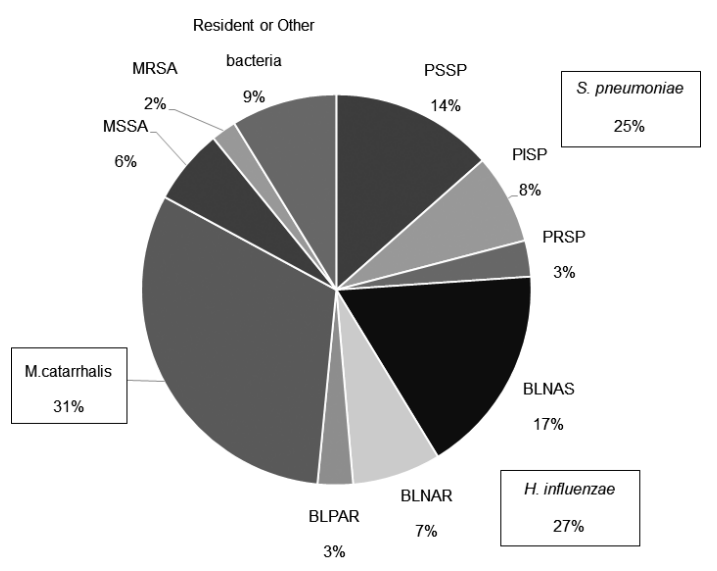

Fig. 1. Breakdown of bacterial species and strains isolated from the nasopharynx of study participants.

Pie chart of the proportions of bacterial species and strains isolated from the nasopharynges of patients under 6 years of age who were hospitalized for lower respiratory tract infection from 2016 to 2018. No strains were isolated from 10 patients ; 1,676 strains were isolated from the remaining 1,046 patients. Eighty-three percent of the bacteria isolated from the nasopharynx were one of the three major causative agents.

PSSP : penicillin-susceptible Streptococcus pneumoniae ; PISP : penicillin-intermediately resistant S. pneumoniae; PRSP : penicillin-resistant S. pneumoniae ; BLNAS : $\beta$-lactamasenonproducing, ampicillin-susceptible Haemophilus influenzae ; BLNAR : $\beta$-lactamase-nonproducing, ampicillin-resistant $H$. influenzae ; BLPAR : $\beta$ lactamase-producing, ampicillin-resistant $H$. influenzae ; MSSA : methicillin-sensitive Staphylococcus aureus; MRSA : methicillin-resistant $S$. aureus strains, of which 226 (14\%) were PSSP, 125 (8\%) were PISP, and $50(3 \%)$ were PRSP. Similarly, $H$. influenzae comprised 463 (27\%) of the isolated strains, of which 291 (17\%) were BLNAS, 123 (7\%) were BLNAR, and 49 (3\%) were BLPAR. $M$. $c a$ tarrhalis accounted for 525 (31\%) of the isolated strains, methicillin-sensitive $S$. aureus comprised 105 (6\%) strains, MRSA comprised 35 (2\%) strains, and resident and other bacteria comprised 147(9\%) strains.

Clinical background of patients from whom one of the three major causative pathogens were isolated

At least one of the three major causative pathogens was isolated from 874 children; the clinical backgrounds and clinical courses of these children are described below. The median age was 14 months ; of the 874 children, $52 \%$ were boys, $75 \%$ had cohabiting siblings, $63 \%$ used daycare, $12 \%$ had underlying diseases, $84 \%$ had received three or more doses of Hib and PCV7/PCV13 vaccine, and $20 \%$ had received antibacterial drugs before admission. The median duration of hospitalization was 5 days and the median duration of post-hospitalization fever was 1 day ; moreover, $76 \%$ of children used antibacterial drugs during hospitalization, $1 \%$ of children received oxygen, and $5 \%$ of children received hydrocortisone.

\section{Analysis of clinical background and course factors ac- cording to isolation of antibiotic-resistant bacteria}

Of the 874 patients from whom at least one of the three major causative pathogens was isolated, antibiotic-resistant bacteria (PISP, PRSP, BLNAR, or BLPAR) were isolated from 328 patients; antibiotic-resistant bacteria were not isolated from 546 patients (Table 1). Univariate analyses revealed that the patients from whom antibiotic-resistant bacteria were isolated were more likely to use daycare and had higher CRP values at admission, compared with patients from whom antibiotic-resistant bacteria were not isolated. A history of three or more $\mathrm{Hib}$ and PCV7/PCV13 vaccinations also tended to be associated with increased isolation of resistant bacteria, although this difference was not statistically significant. In the multivariate analysis, daycare use was the only significant predictor for isolation of antibiotic-resistant bacteria. 
Table 1. Factors associated with patients' background and clinical course : PSSP, BLNAS, or M. catarrhalis isolates vs PISP, PRSP, BLNAR, or BLPAR isolates

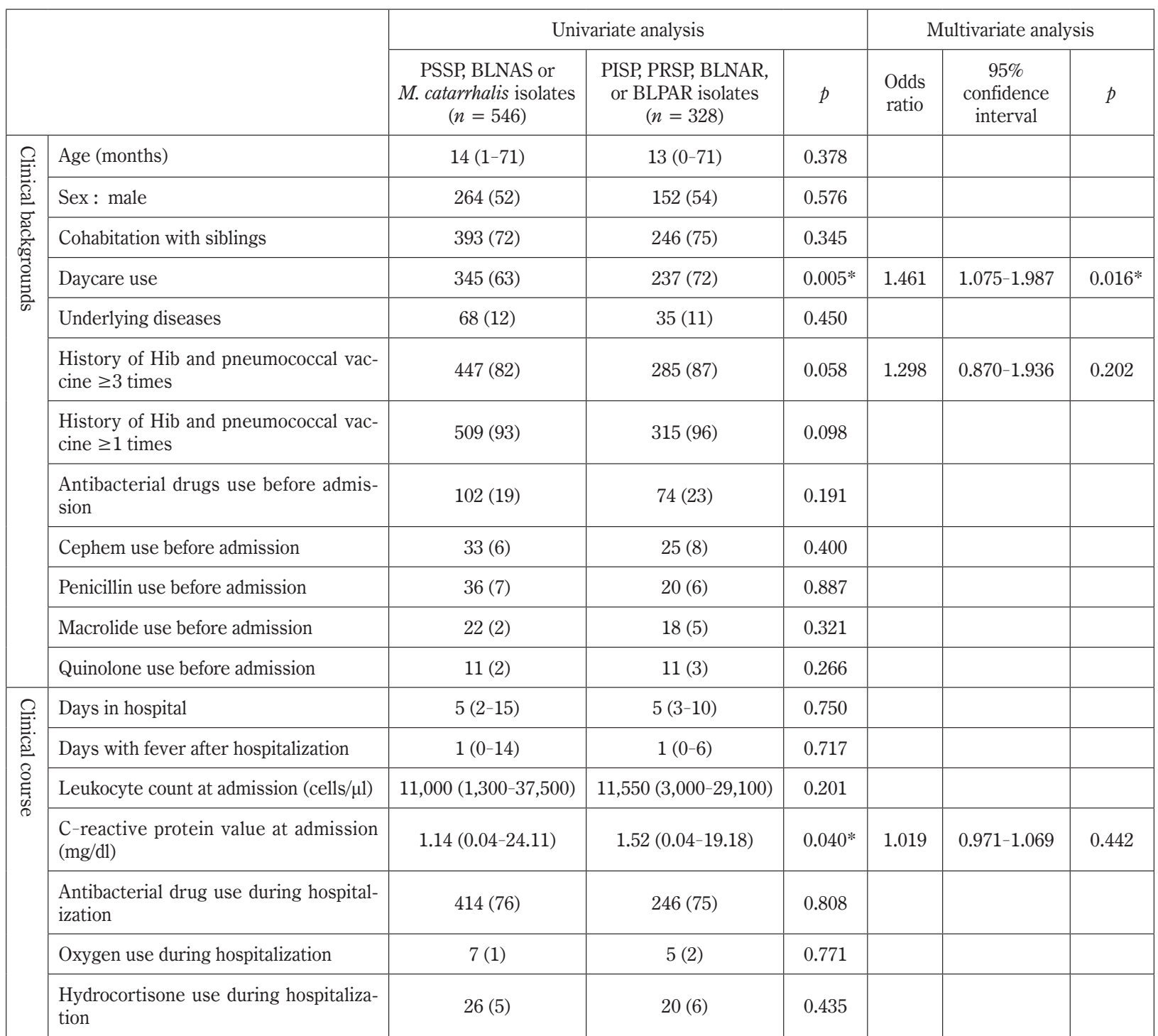

The chi-square test was used to compare categorical variables, shown as $n(\%)$; the Mann-Whitney $\mathrm{U}$ test was used to compare continuous variables, shown as median (range).

Logistic regression analysis was performed for the multivariate analysis ; each factor with a $p$ value of $<0.05$ was used as an independent factor for the univariate analysis.

*Statistically significant values.

Hib : Haemophilus influenzae type b vaccine; pneumococcal vaccine : pneumococcal conjugate vaccines 7 and 13 ; PSSP : penicillin-susceptible Streptococcus pneumoniae ; PISP : penicillin-intermediately resistant Streptococcus pneumoniae ; PRSP : penicillin-resistant Streptococcus pneumoniae ; BLNAS : $\beta$-lactamase-nonproducing, ampicillinsusceptible Haemophilus influenzae; BLNAR : $\beta$-lactamase-nonproducing, ampicillin-resistant Haemophilus influenzae ; BLPAR : $\beta$-lactamase-producing, ampicillin-resistant Haemophilus influenzae

\section{Discussion}

We investigated the nasopharyngeal culture on admission in children with lower respiratory tract infection and found that $99 \%(1,046$ of 1,056) of these children had positive bacterial culture results ; either S. pneumoniae, H. influenzae, or M. catarrhalis was isolated from $83 \%$ (874 of 1,046) of them.
Definitive methods for diagnosing the causative bacteria of pneumonia include isolation of bacteria from blood, pleural effusion, or lung puncture ; however, these invasive methods were hardly suitable for the children in this study. In practice, transtracheal aspirations are routinely collected from intubated children and sputum from the pharynx is collected from non-intubated children for washed 
sputum culture $^{6)}$. As a practical matter, it is quite difficult to collect sputum from infants. The nasopharyngeal swab sample collection applied in this study has the advantage of noninvasive and reliable sampling. A matched case-control study demonstrated high concordance between nasopharyngeal and endotracheal aspirate samples or bronchoalveolar lavage fluid samples; thus, the authors presumed that nasopharyngeal microbiota can serve as a valid proxy for lower respiratory tract microbio$\mathrm{ta}^{7)}$. In contrast, another report indicated low concordance between isolates from nasopharyngeal swabs and bronchoalveolar fluid ${ }^{8)}$. Because it is controversial whether the nasopharyngeal microbiota can reflect the lower respiratory tract microbiota, nasopharyngeal culture results should be collected as a reference finding ; sputum and tracheal aspirate cultures should be performed as needed, especially in patients with severe respiratory infection.

S. pneumonia, $H$. influenzae, and $M$. catarrhalis, the three species most often isolated from the nasopharynx among patients in this study, are bacteria that colonize the nasopharynx early in life ${ }^{9)}$; thus, it is unlikely that all isolated bacteria were pathogenic. However, the isolation rate of these bacteria is reportedly higher in children with upper respiratory tract infections than in healthy children ${ }^{10,11)}$. Bacteria that proliferate in the upper respiratory tract after airway inflammation triggered by viral infection may also influence the development of lower respiratory tract inflammation by mechanisms such as entry into the lower respiratory tract and aspiration of airway secretions ${ }^{12,13)}$. Accordingly, the nasopharyngeal microbiota is presumed to affect the onset of both upper and lower respiratory tract infections ${ }^{9)}$. Furthermore, recent 16S RNA profiling-based analysis of the nasopharyngeal microbiota has revealed an association with subsequent wheezing ${ }^{14,15)}$. Because of changes in environmental factors and their mutual relationship to the host, the nasopharyngeal microbiota presumably comprises both resident species and species involved in the development of lower respiratory tract infection ${ }^{7,16)}$.

Regarding the involvement of $S$. aureus, our previous study showed that the number of children with isolates of the three major causative pathogens and the number of children with isolates of $S$. aureus or other bacteria were almost equal among older children (i.e., $\geq 4$ years of age ; data not shown). Multivariate analysis of factors associated with clinical severity between these two groups did not identify any statistically significant factors (data not shown). Thus, we concluded that the clinical impact of $S$. $a u$ - reus or other bacteria on lower respiratory tract infection is low ; additionally, the isolation rates of these bacteria were lower in patients under 6 years of age in the present study. Accordingly, we focused on children from whom at least one of the three major causative pathogens had been isolated.

Further analysis in this study comprised examination of the clinical backgrounds and clinical course of patients in whom antibiotic-resistant bacteria were isolated from the nasopharynx. A multivariate analysis of all patients revealed that daycare use was the only significant predictor of antibiotic-resistant bacteria isolation. In the field of otolaryngology, it has been reported that children with recurrent otitis media frequently carry resistant bacteria ${ }^{17)}$. However, children with resistant strains of bacteria do not necessarily exhibit refractory or recurrent otitis media ; they are often asymptomatic ${ }^{18)}$. Daycare facilities are densely populated and prone to droplet infection, and then may promote the spread of bacteria. Children recovering from upper respiratory tract infections are likely to be repeatedly infected by surrounding healthy carriers, leading to a high incidence of resistant strains ${ }^{17)}$. The present study which investigated nasopharyngeal bacteria in preschool children, confirmed the association between daycare use and the transmission of antibiotic-resistant bacteria. These findings imply that daycare users should refrain from attending daycare facilities when they are symptomatic; daycare staff should perform thorough infection control measures.

In univariate analyses of clinical factors, the isolation of antibiotic-resistant strains was higher among patients who had received the Hib and pneumococcal vaccines, but this factor was not statistically significant in the multivariate analysis. This may be due to the high vaccination and hospital visit rates of patients using daycare facilities.

In this study, the isolation of antibiotic-resistant bacteria was not associated with factors related to the severity of lower respiratory tract infection (i.e., hospital stay length ; period of post-hospitalization fever ; or administration of antibacterial agents, oxygen, or hydrocortisone). For patients with lower respiratory tract infection who were treated with antibacterial agents, the need for such treatment was determined by comprehensively considering the clinical symptoms, laboratory findings, and clinical course. Piperacillin (2016-2017) or ampicillin (2018) was usually selected as the initial drug for treating lower respiratory tract infection ; its selection was not based on an assumption of antibioticresistant bacteria. Because we found no association 
between the antibiotic resistance of nasopharyngeal bacteria and severity of lower respiratory tract infection, we recommend against using the results of nasopharyngeal culture as a basis for antibiotic selection or modification. For the initial selection of antibiotics to treat lower respiratory tract infection, our findings support the selection of narrow-range antibiotics without considering the possibility of antibiotic-resistant strains, except in patients with serious systemic infections that yield positive blood culture findings.

This study had a few limitations. First, virological examination of the patients was insufficient. Rapid antigen tests were performed in the patients according to the epidemic season, but not in all cases, and detection by polymerase chain reaction or other methods was not performed. Virus infection may be involved in the development of lower respiratory tract infection. In other reports, viruses and bacteria were simultaneously detected in the nasopharynx of children with lower respiratory tract infections ${ }^{19-21)}$, and their interaction is assumed. Further studies are needed to evaluate the relationship between viral infections and resistant bacteria. Second, evaluations of the presence or absence of otitis media and sinusitis, blood culture, and antibody tests were not performed in some patients ; moreover, some bacterial infections were insufficiently evaluated. Blood culture was performed for analysis of patients whose admission blood tests revealed a highly inflammatory reaction, but there were no patients with positive blood culture results who exhibited invasive bacterial infection.

This study suggests that the increased detection rate of resistant bacteria may be related to daycare use. In many instances, the isolation of such bacteria does not affect the severity of lower respiratory tract infection. Therefore, if antibacterial agents are used in the initial treatment of lower respiratory tract infection, narrow-range antibacterial agents should be selected in accordance with current guidelines for treatment of respiratory diseases in children.

\section{Acknowledgments}

We would like to express our deep gratitude to the staff of the clinical laboratory department and pediatric ward of Soma General Hospital for their cooperation in this research. We also thank Katie Oakley, $\mathrm{PhD}$ and Ryan Chastain-Gross, PhD from Edanz (https://jp.edanz.com/ac) for editing a draft of this manuscript.
The abstract of this paper was presented at the $51^{\text {st }}$ Annual Meeting of the Japanese Society of Pediatric Infectious Diseases (October 2019, Asahikawa).

\section{Conflict of interest disclosure}

None to declare

\section{References}

1. Sectish TC, Prober CG. Pneumonia. In : Kriegman Rm, Behrman RE, eds. Nelson textbook of pediatrics. 18th ed. Saunders, Philadelphia, 1795$1800,2007$.

2. Nohynek H, Eskola J, Kleemola M, Jalonen E, Saikku P, Leinonen M. Bacterial antibody assays in the diagnosis of acute lower respiratory tract infection in children. Pediatr Infect Dis, 14 : 478484, 1995.

3. Borges IC, Andrade DC, Vilas-Boas AL, et al. Detection of antibody responses against Streptococcus pneumoniae, Haemophilus influenzae, and Moraxella catarrhalis proteins in children with community-acquired pneumonia : effects of combining pneumococcal antigens, pre-existing antibody levels, sampling interval, age, and duration of illness. Eur J Clin Microbiol Infect Dis, 34 : 1551-1557, 2015.

4. Sasaki K, Sato E, Saitoh N, Komatsu A. Bacteriological investigation of nasopharyngeal culture in the pediatric department in our hospital. (in Japanese) Japanese Journal of Medical Technology, 59 : 1292-1296, 2010.

5. Sakwinska O, Bastic Schmid V, Berger B, et al. Nasopharyngeal microbiota in healthy children and pneumonia patients. J Clin Microbiol, 52 : 1590-1594, 2014.

6. Uehara S, Sunakawa K, Eguchi H, et al. Japanese Guidelines for the Management of respiratory tract infectious diseases in children 2007 with focus on pneumonia. Pediat Int, 53 : 264-276, 2011.

7. Man WH, Houten MA, Mérelle ME, et al. Bacterial and viral respiratory tract microbiota and host characteristics in children with lower respiratory tract infections: a matched case-control study. Lancet Respir Med, 7 : 417-426, 2019.

8. Escribano Montaner A, García de Lomas J, Villa Asensi JR, et al. Bacteria from bronchoalveolar lavage fluid from children with suspected chronic lower respiratory tract infection : results from multi-center, cross-sectional study in Spain. Eur J Pediatr, 177 : 181-192, 2018.

9. Hotomi M, Yamanaka N. Dynamism of nasopharyngeal bacterial flora. (in Japanese) Stomatopharyngol, 23 : 59-63, 2010. 
10. Faden H, Duffy L, Wasielewski R, Wolf J, Krystofik D, Tung Y. Relationship between nasopharyngeal colonization and the development of otitis media in children. J Infect dis, 175 : 140-145, 1997.

11. Ito M, Ito K, Yoshizaki T, Nishimura T, Miwa T, Furukawa M. Nasopharyngeal penicillin-resistant Streptococcus pneumoniae strains among young children in Japan. Otol Neurotol, 23 : 349-352, 2002.

12. Takase H, Nitanai H, Yamamura E, Otani T. Facilitated expansion of pneumococcal colonization from the nose to the lower respiratory tract in mice preinfected with influenza virus. Microbiol Immunol, 43 : 905-907, 1999.

13. Marik PE. Aspiration pneumonitis and aspiration pneumonia. N Engl J Med, 344 : 665-671, 2001.

14. Teo SM, Tang HHF, Mok D, et al. Airway Microbiota Dynamics Uncover a Critical Window for Interplay of Pathogenic Bacteria and Allergy in Childhood Respiratory Disease. Cell Host Microbe, 24 : 341-352, 2018.

15. McCauley K, Durack J, Valladares R, et al. Distinct nasal airway bacterial microbiotas differentially relate to exacerbation in pediatric patients with asthma. J Allergy Clin Immunol, 144 : 11871197, 2019.

16. Bosch AATM, de Steenhuijsen Piters WAA, van Houten MA, et al. Maturation of the Infant Respi- ratory Microbiota, Environmental Drivers, and Health Consequences. A Prospective Cohort Study. Am J Respir Crit Care Med, 196 : 15821590, 2017.

17. Nagata R. Examination of the resistance trend of nasopharynx origin streptococcus pneumoniae in a normal child and adult (Examination of nasopharynx bacterial flora in consideration of an infectious disease treatment strategy). (in Japanese) J Juzen Med Soc, 115 : 75-85, 2006.

18. Yamanaka N, Hotomi M. Molecular-biological and immunological approaches against intractable acute otitis media in children. (in Japanese) The Journal of the Japanese Association of Infectious Diseases, 77 : 595-605, 2003.

19. Tenenbaum T, Franz A, Neuhausen N, et al. Clinical characteristics of children with lower respiratory tract infections are dependent on the carriage of specific pathogens in the nasopharynx. Eur J Clin Microbiol Infect Dis, 31 : 3173-3182, 2012.

20. Juvén T, Mertsola J, Waris M, et al. Etiology of community-acquired pneumonia in 254 hospitalized children. Pediatr Infect Dis J, 19 : 293-298, 2000.

21. Liu P, Xu M, He L, et al. Epidemiology of Respiratory Pathogens in Children with Lower Respiratory Tract Infections in Shanghai, China, from 2013 to 2015. Jpn J Infect Dis, 71 : 39-44, 2018. 